\title{
Temperature, Strain and Strain Rate Dependence of Serrated Flow in Bulk Metallic Glasses
}

\author{
Florian H. Dalla Torre*1, Alban Dubach, Adrienne Nelson*2, Jörg F. Löffler \\ Laboratory of Metal Physics and Technology, Department of Materials, ETH Zurich, \\ Wolfgang-Pauli-Str. 10, 8093 Zurich, Switzerland
}

\begin{abstract}
New findings on the inhomogeneous plastic deformation of bulk metallic glasses (BMGs) have shown a disappearance of serrated flow below a critical temperature or above a critical strain rate. This correlates with a change in the strain rate sensitivity (SRS) from negative to positive values, suggesting a change in the deformation mechanism. In addition, a change in the SRS correlating with the increase in the stress drop magnitude is observed with increasing strain. Results on the serration and flow dependence for the binary $\mathrm{BMG} \mathrm{Cu}_{50} \mathrm{Zr}_{50}$ show close phenomenological similarities with the Portevin - Le Châtelier or dynamic strain aging effect known for crystalline solids. An alternative model for the appearance and disappearance of serrated flow based on the structural relaxation of the atomic configuration of shear transformation zones is described. [doi:10.2320/matertrans.MJ200782]
\end{abstract}

(Received December 19, 2006; Accepted January 22, 2007; Published June 25, 2007)

Keywords: bulk metallic glasses, serrated flow, deformation kinetics, shear band relaxation, strain rate sensitivity

\section{Introduction}

With respect to mechanical properties, amorphous metals distinguish themselves from their coarse-grained crystalline counterparts through their extraordinary high strength but limited ductility at room temperature. ${ }^{1-4)}$ Recently a few publications have attracted considerable attention due to the large plastic strain in compression reported for a number of bulk metallic glasses (BMGs) ${ }^{5-10)}$ Several explanations have been put forward to explain such large compressive strains: (i) a high Poisson's ratio or low bulk modulus typically found in noble metals, which cause a toughening of the material; $;$,11) (ii) dispersion of nanocrystals and/or chemically different phases embedded in the amorphous matrix, which result in the splitting and multiplication of shear bands; ${ }^{6,7)}$ or (iii) in-situ-deformation-induced nanocrystallization processes, which prevent strain softening and cause a delocalising of the stress field. ${ }^{9,10,12)}$ These observations give a clearer picture of the deformation behavior, microstructural influences and changes required for an increase in plastic strain and the shear banding processes of metallic glasses in general. However, a detailed microscopic, kinetic and thermodynamic interpretation of the shear process is still pending.

Based on Argon's concept ${ }^{13)}$ of shear transformation zones (STZs) as the carrier of plastic deformation in metallic glasses, more refined thermodynamic considerations of the shear process have recently been proposed. ${ }^{14)}$ Parameters such as the strain rate sensitivity (SRS), size of the activated volume in a STZ and potential energy of deformation have also been derived recently from computer simulations. ${ }^{15-18)}$ These results combined with Refs. 13 and 14 have provided a deeper understanding of the kinetics of STZs - information which is difficult to assess experimentally.

Many early results on metallic glasses provide important

\footnotetext{
${ }^{* 1}$ Corresponding author, E-mail: florian.dallatorre@mat.ethz.ch

*2Permanent address: Department of Materials Science and Metallurgy,

University of Cambridge, Pembroke Street, Cambridge CB2 3QZ, UK
}

information for the characterization of inhomogeneous flow behavior in amorphous metals. ${ }^{19-22)}$ One of the first experiments tackling the inhomogeneous flow kinetics in metallic glasses has shown preferential etching of shear bands in deformed samples - a clear indication of a modification of the atomic structure during straining, ${ }^{19)}$ as was also suggested recently in Refs. 23-25. Serrated flow was recognized in stress-strain diagrams, and each measured stress drop correlated with a shear event. It was also noted that preexisting shear bands are reactivated more easily than new shear bands are formed, which is further evidence for the change in the atomic structure of shear bands. ${ }^{19,20)}$ In addition, a change in the serration amplitude with increasing strain was observed and associated with different stages of deformation. ${ }^{20)}$ The temperature dependence of the appearance and the disappearance of serrations observed in a stress strain diagram ${ }^{19-22)}$ was explained with an adiabatic shear model proposed by Chen and Leamy. ${ }^{20,26)}$ In this model a sufficiently high temperature is needed for the shear event to initiate a local decrease in viscosity and result in an overall drop in the stress, i.e. serrated flow. ${ }^{20)}$

More recent studies on the mechanical flow behavior of metallic glasses have mainly been performed by nanoindentation, ${ }^{27-33)}$ where instabilities and early failure are not as critical as in compression or bending. In agreement with studies on other deformation modes, ${ }^{22,34,35)}$ these investigations reveal a decrease in the serration amplitude ('pop-ins') with increasing indentation rate. The tests indicate that shear band formation not only causes a sudden decrease in the load, i.e. local elastic stress drop, but actively contributes to plastic deformation by a shear offset of up to several tens of nanometers. Based on the observed disappearance of 'popins', which means a transition from discrete to continuous shearing, Schuh et al. ${ }^{31)}$ proposed a new homogeneous deformation regime which is kinetically driven by the superior rate of deformation compared to the rate of shear band nucleation. This simultaneous operation of multiple shear bands at higher rates leads to a spatially more homogeneous flow. ${ }^{30)}$ In agreement with Schuh's work, ex- 
situ and in-situ indentation studies coupled with scanning electron microscopy (SEM) have shown fewer shear bands with larger offsets for smaller indentation rates and a transition from discrete to gradual shear band formation with increasing loading rates. ${ }^{29,33)}$ In contrast to Ref. 20 the load drop/displacement burst occurring during shear band formation has been rationalized by STZs, which percolate to an infinitely fast-running shear band once a critical number of nuclei has been activated. ${ }^{28)}$

In compression test experiments performed by Wright et al. ${ }^{36)}$ where a data acquisition rate of $100 \mathrm{~Hz}$ was applied, the serrated flow and its associated stress drop amplitude were investigated. In addition, the adiabatic heat generated by a single shear event was estimated by evaluating the temporal resolution of a stress drop - a topic that has recently attracted great scientific interest (see e.g. ${ }^{37)}$ ). According to Refs. 36, 38 the temperature increase due to adiabatic heating is not sufficient to cause a local drop in the viscosity and, therefore, cannot explain serrated flow behavior of shear banding, in particular, with regard to the shear band thickness. This conclusion contrasts with that of a recent experiment ${ }^{37)}$ where a tin coating on a metallic glass was used as a thermometer for shear banding. Since molten droplets of pure tin were found at the position of the shear bands it was concluded that (as assumed in Refs. 20, 26) locally and temporally very high temperatures of several hundred degrees can be reached and may effect a change in the local atomic structure within shear bands. This conclusion also provides a plausible explanation for the observed straininduced nanocrystallization..$^{9,10,12,39)}$

Conceptually, our work builds on the early experiments undertaken in Refs. 19, 20, 22. As we have shown recently for bulk amorphous $\mathrm{Zr}_{52.5} \mathrm{Ti}_{5} \mathrm{Cu}_{17.9} \mathrm{Ni}_{14.6} \mathrm{Al}_{10}(\mathrm{Vit} 105)^{35,40)}$ the tests performed provide a new insight into the characteristics of serrated flow as a function of strain, strain rate, and temperature. These parameters are of great importance for the understanding of shear banding in metallic glasses. We also correlate the disappearance and appearance of serrated flow with a change in the strain rate sensitivity and highlight the phenomenological similarities of shear banding in metallic glasses with that in crystalline solid solution metals. The latter is known to be caused by the dynamic strain aging (DSA) effect ${ }^{41,42)}$ and was originally observed as shear bands by Portevin and Le Châtelier. ${ }^{43}$

\section{Experimental Procedure}

$\mathrm{Zr}_{50} \mathrm{Cu}_{50}$ prealloys were prepared by arc melting the pure elements (purity $>99.995 \%$ ) in a Zr-gettered argon atmosphere from which cylindrical rods of $2 \mathrm{~mm}$ in diameter and $\sim 30 \mathrm{~mm}$ in length were suction cast or drop cast by levitation into a copper mold. Compression test specimens with a length-to-diameter ratio of $2: 1$ or $5: 3$ were cut from these rods and both ends were polished to ensure parallelism of the compression planes. The amorphous and/or crystalline structure of the specimens was confirmed by means of $\mathrm{X}$ ray diffraction (XRD) using a PANalytical X'pert diffractometer with $\mathrm{Cu}-K_{\alpha}$ radiation or a Rigaku diffractometer equipped with a Co anode, and by differential scanning calorimetry (DSC) using a Setaram Labsys DSC. The fractured specimens were examined with a scanning electron microscope (Leo 1530). Monotonic and cycled strain rate tests in compression were performed on a screw-driven testing machine (Schenck Trebel) over a temperature range of $77 \mathrm{~K}$ to $300 \mathrm{~K}$, where strain was measured from the crosshead displacement and with a clip gauge mounted on the pistons directly above and below the specimen. Due to the stiffness of the machine-specimen assembly this mode of strain measurement typically results in lower elastic modules or larger elastic strain limits compared to strain measurements performed with a clip gauge directly mounted onto the specimen. As reported earlier elastic strain limits of $2 \%$ can be expected for the $\mathrm{Cu}_{50} \mathrm{Zr}_{50}$ alloy. ${ }^{6,8)}$ An acquisition rate of 20 to $300 \mathrm{~Hz}$ was used, depending on the detail of information needed. (The load cell response yields a resolution of about 2-3 MPa for the slowest acquisition rate.) Cyclic strain rate jump tests were performed by alternating the crosshead speed between two distinct values once the flow stress of the material was reached. Typically a ratio of $\dot{\varepsilon}_{2} / \dot{\varepsilon}_{1}=10$ was chosen, and the applied strain rates ranged from $3.33 \times 10^{-5} \mathrm{~s}^{-1}$ to $0.2 \mathrm{~s}^{-1}$. The asymptotic strain rate sensitivity was evaluated from the two different steady state stress responses caused by the strain rate change after the transients took place (for more details see Ref. 35). The samples were cooled to the desired temperature between $77 \mathrm{~K}$ (liquid nitrogen) and room temperature by immersing them in various cooling mixtures with ethanol and liquid nitrogen or dry ice $\left(\mathrm{CO}_{2}\right)$ during testing.

\section{Results}

\subsection{Influence of crystal fraction on the mechanical properties}

Figure 1(a) shows XRD scans of three different representative $\mathrm{Cu}_{50} \mathrm{Zr}_{50}$ samples of $2 \mathrm{~mm}$ diameter measured with the Co- $K_{\alpha}$ source. Not all of the produced samples show a fully amorphous microstructure. Some crystalline peaks can be observed in the XRD scans overlaying the amorphous hump. Variations in the fraction of crystals embedded in the amorphous matrix were measured when comparing different rods as well as when comparing measured slices cut from different positions in the same rod. This inhomogeneity in the microstructure reflects the sensitivity to glass-forming ability of the binary alloy and the critical casting thickness, previously reported to lie between 1 to $2 \mathrm{~mm} \mathrm{[6,8}$ and refs. therein].

Mechanical properties are greatly affected by the fraction of crystals embedded in the amorphous matrix. This is shown in Fig. 1(b), where the stress-strain curve of a fully amorphous sample ' $A$ ' is shown together with that of a sample 'B', which contains a certain fraction of crystals in the amorphous matrix. While sample ' $A$ ' shows a sharp elastic/plastic transition, no strain hardening (perfect plastic behavior) and visible serrations, sample ' $\mathrm{B}$ ' shows a lower yield point, significant strain hardening, and little to no serrated flow because of the dislocation-dominated deformation in the crystals. Thus only those samples which show properties similar to ' $A$ ' were regarded as valid for our analysis of the mechanical behavior of bulk amorphous $\mathrm{Cu}_{50} \mathrm{Zr}_{50}$. 

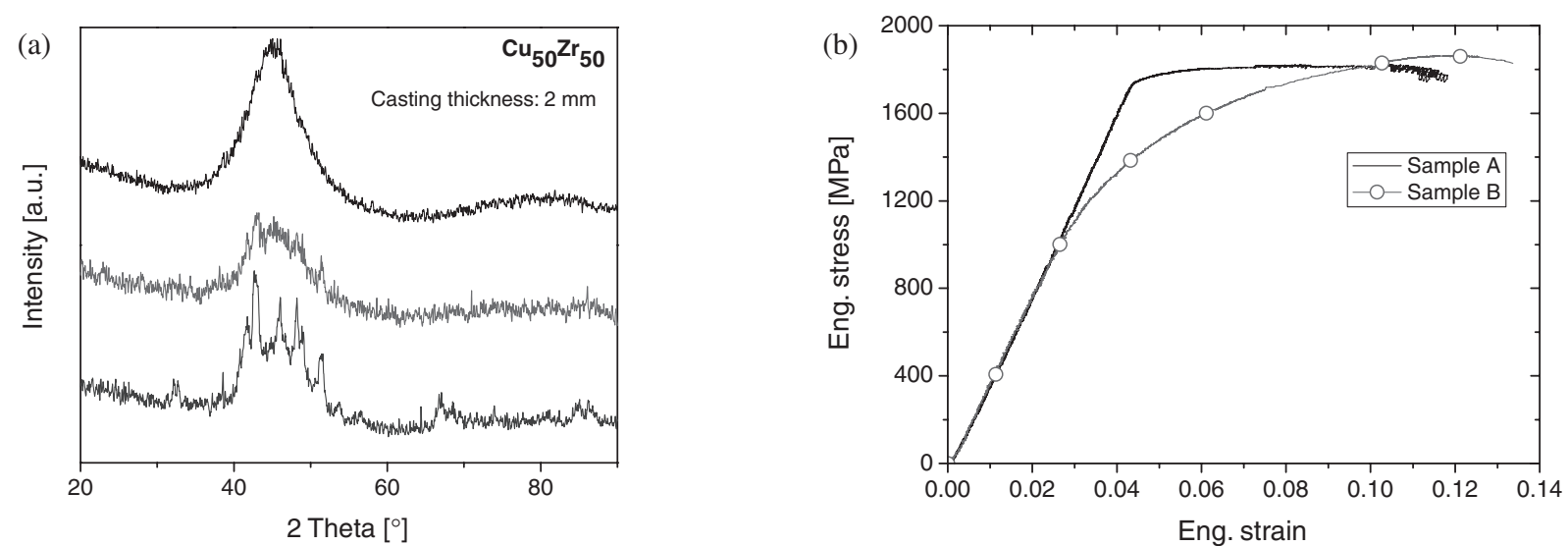

Fig. 1 (a) XRD scan of different slices cut from $2 \mathrm{~mm}$ rods of $\mathrm{Cu}_{50} \mathrm{Zr}_{50}$; (b) stress-strain curves of an amorphous (sample A) and partiallyamorphous (sample B) specimen.


Fig. 2 (a) Stress-strain curve recorded at initial strain rate of $1.0 \times 10^{-3} \mathrm{~s}^{-1}$ at a data-acquisition rate of $50 \mathrm{~Hz}$. The inset shows the increase in stress drop magnitude with increasing strain; (b) closeup of two areas of the stress strain curve shown in (a) (rectangular areas) indicating the temporal resolution of shear band events. Neighboring data points are $6.67 \mathrm{~ms}$ apart.

\subsection{Serrated flow at room temperature}

Figure 2(a) shows a stress-strain curve of a specimen tested at room temperature and at an initial strain rate of $1.0 \times 10^{-3} \mathrm{~s}^{-1}$. The inset shows the magnitude of stress drops in the course of deformation. A clear increase in the stress drops from $\sim 5 \mathrm{MPa}$ at the beginning of deformation to values close to $30 \mathrm{MPa}$ towards the end of deformation was recorded. A lower limit of $5 \mathrm{MPa}$ was used to clearly differentiate the serration amplitude from the noise in the range of 2-3 MPa. In this context it should be noted that these stress drops are understood as the lower limit of the effective stress drops occurring in the sample. It was shown by Kimura and Masumoto ${ }^{21)}$ that the damping effects of the machine assembly, typically with load cells which are far from the specimens, substantially lower the ability to capture the dynamic stress response which occurs during a serration event. Applying their evaluation method to the effective stress drops in the machine assembly used in this study yields a stress drop per shear event 2 to 3 times larger than that measured and shown in Fig. 2(a). Therefore, effective stress drops close to $100 \mathrm{MPa}$ have to be envisioned and supported by the material in order to prevent fracture!

Figure 2(b) shows two regions, designated as rectangles in the stress-strain curve of Fig. 2(a), plotted as a function of strain and time. The curve was recorded at an acquisition rate of $150 \mathrm{~Hz}$, yielding a data-point (open squares) every $6.67 \mathrm{~ms}$. In the lower strain range after $\sim 30$ seconds small stress drops are measured, which in some cases show several data points on the downward branches of the serrations. The drop times measured lie between 10 and 100 $\mathrm{ms}$. In the larger strain range, after $\sim 40$ seconds significantly larger stress drops are captured which are recorded between a few data points only, resulting in stress drop times of approximately $10 \mathrm{~ms}$. Our results thus provide evidence that not only does the amplitude increase with increasing strain, but that also the speed of the stress drops (that is, the speed of a shear band event) increases with increasing deformation. In addition, we observed, within the same strain range for the various samples analysed, a decrease in the stress drop magnitude with increasing strain rate: from $\sim 15 \pm 10 \mathrm{MPa}$ at strain rates of $\sim 10^{-4} \mathrm{~s}^{-1}$ to $\sim 8 \pm 5 \mathrm{MPa}$ at $\sim 10^{-3} \mathrm{~s}^{-1}$. The same trend, but with somewhat larger stress amplitudes, was also observed in Vit105. ${ }^{35)}$ In addition, a decrease in the time needed for a stress drop or shear event to initiate, propagate and become arrested was observed with increasing strain rate. Whether 

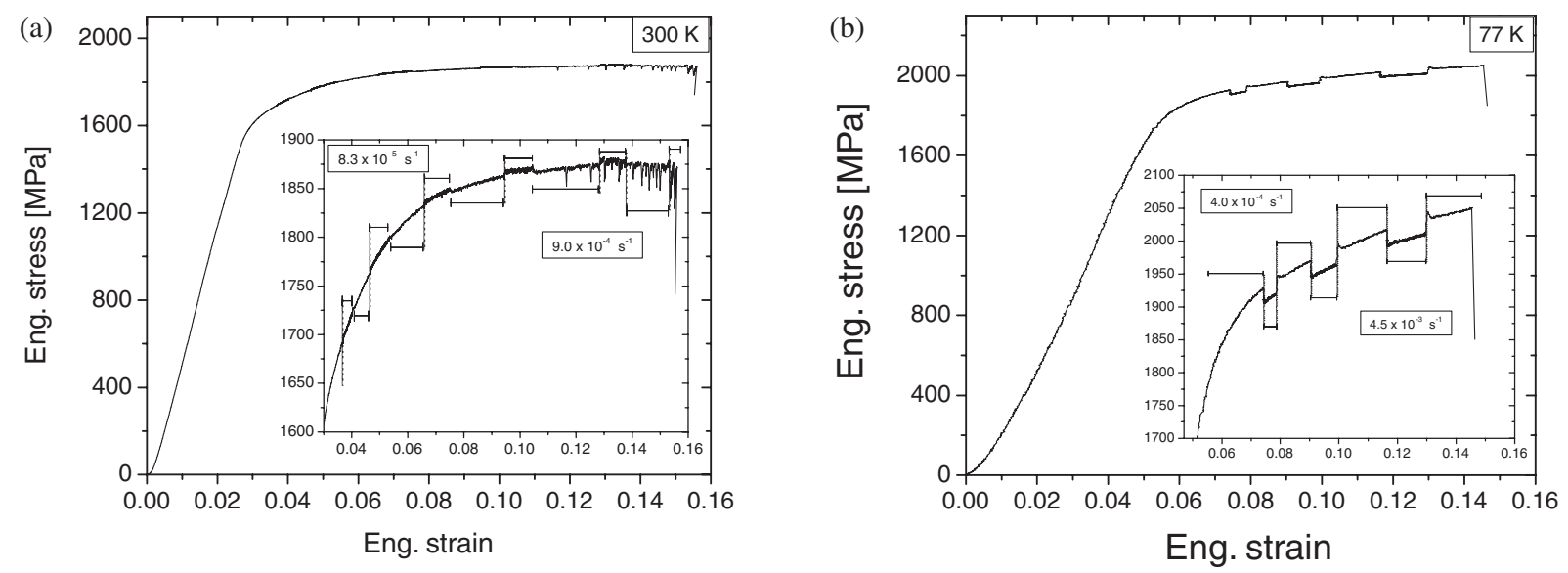

Fig. 3 Stress-strain curves of $\mathrm{Cu}_{50} \mathrm{Zr}_{50}$ tested at strain rates alternating between $10^{-4}$ and $10^{-3} \mathrm{~s}^{-1}$ at $300 \mathrm{~K}$ (a) and $77 \mathrm{~K}$ (b). While in (a) the strain was measured by the strain gauge mounted close to the specimen, this was not possible at low temperatures, so that a linear inductive displacement transducer mounted further away from the specimen had to be used. This causes a lower elastic slope due to the larger contribution of the machine compliance.

this is due to the smaller drops at higher strain rate or whether the shear events are faster is, however, unclear.

\subsection{Strain, strain rate and temperature sensitivity of inhomogeneous flow}

Figure 3 shows stress-strain curves measured at room temperature (Fig. 3(a)) and liquid nitrogen temperature (Fig. 3(b)) using alternating strain rates. In Fig. 3(a) an increase in the strain rate by one order of magnitude causes a drop of the stress in the range of a few MPa's. As shown in the inset, not only the upper boundary of the serrated flow curves responds to a change in strain rate, but also the lower part of the curve, where serrations are arrested. This observation agrees with that made by Chen for Pd-based metallic glasses $\left(\mathrm{Pd}_{77.5} \mathrm{Cu}_{6.0} \mathrm{Si}_{16.5}\right),{ }^{20)}$ however, his conclusion that this behavior is different from that seen in crystalline alloys, where serrated flow is caused by the dynamic strain aging effect resulting in Portevin-Le Châtelier bands, has been shown to be incompatible with more recent observations. ${ }^{44-46)}$ Different serrations (type A, B, and C) can also be distinguished in these crystalline alloys, which indeed have similar strain and strain rate dependencies as observed in this study.

As shown in Fig. 3(b) at liquid nitrogen temperature, a smooth yielding occurs without any sign of serrated flow. In addition, an increase in the strain rate by one order of magnitude yields a significant increase in strength of a few tens of MPa's. As mentioned in Section 1, the disappearance of serrated flow at low temperatures was observed earlier, ${ }^{19,20,22)}$ but was not correlated with a change in the strain rate sensitivity (see below) and was also not taken into account in more recent studies.

Generally, a change in the applied strain rate $\dot{\varepsilon}$ (or cross head speed) causes a change in the stress $(\sigma)$ response of the material for a given strain $(\varepsilon)$. This kinetically-driven response can be quantified by the strain rate sensitivity parameter, $m$, where

$$
m=\left(\frac{\partial \ln \sigma}{\partial \ln \dot{\varepsilon}}\right)_{\varepsilon} .
$$

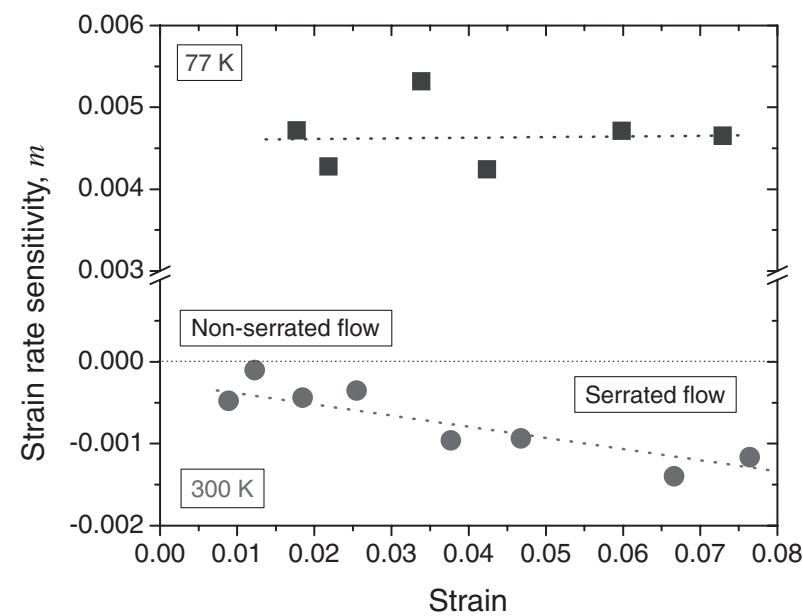

Fig. 4 Strain rate sensitivity as a function of strain for $300 \mathrm{~K}$ and $77 \mathrm{~K}$. The dashed lines are guides for the eye.

Materials exhibiting values of $m$ close to zero are not ratesensitive (typical for athermal deformation mechanisms such as dislocation glide), while a value of unity corresponds to perfect Newtonian viscosity, where the stress is directly proportional to the strain rate (purely thermally activated).

The decrease in the stress with increasing strain rate measured in metallic glasses at room temperature (Fig. 3(a)) reveals a negative SRS, while a positive SRS is measured for tests performed at liquid nitrogen temperatures. This is shown in Fig. 4, where the SRS is plotted against the strain for the tested samples presented in Fig. 3. As shown in Fig. 4, $m$ decreases with increasing $\varepsilon$ at room temperature, while no variation of $m$ on $\varepsilon$ is detected at $77 \mathrm{~K}$. This was also observed for different strain rates measured on various samples.

In Fig. 5 the mean value for $m$ of each tested sample is plotted against the strain rate for plastic strains within $\sim 0.01$ to $\sim 0.1$. A clear decrease in the strain rate sensitivity from 0.008 at the slowest strain rates to values in the range of 0.002 to -0.001 at the highest strain rates are measured at $77 \mathrm{~K}$. A similar, but much less pronounced, decrease in $m$ with increasing $\dot{\varepsilon}$ can also be observed at $153 \mathrm{~K}$. At room 


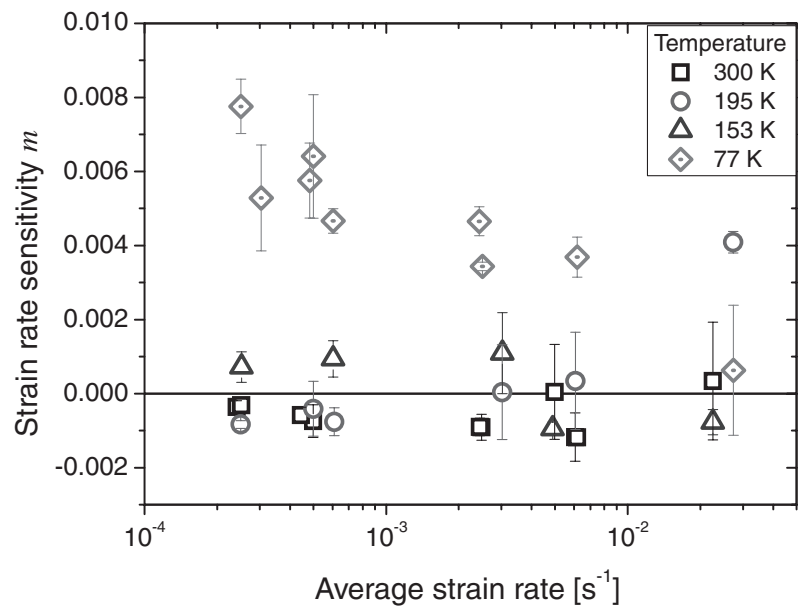

Fig. 5 Mean strain rate sensitivity measured as a function of the average strain rate applied for temperatures of $300,195,153$ and $77 \mathrm{~K}$. Note that at higher strain rates $\left(>10^{-2} \mathrm{~s}^{-1}\right)$ data accuracy is lower due to the high speed of testing, which only allows one to two changes in the strain rate per test.

temperature and $195 \mathrm{~K}$, on the other hand, a change from negative values of $m$ to slightly positive ones was measured at the highest strain rates. At low strain rates hardly any difference in $m$ is measured for tests performed at $300 \mathrm{~K}$ compared to $195 \mathrm{~K}(\Delta T \sim 100 \mathrm{~K})$, while a significantly larger difference in $m$ is measured if tested at $77 \mathrm{~K}$ compared to $153 \mathrm{~K}(\Delta T \sim 75 \mathrm{~K})$ for the same strain rates. At high strain rates, however, the reverse dependence on $m$ has been observed: larger differences in $m$ for $300 \mathrm{~K}$ and $195 \mathrm{~K}$ and smaller difference in $m$ for $77 \mathrm{~K}$ and $153 \mathrm{~K}$. This temperature sensitivity of $m$ at low $\dot{\varepsilon}$ suggests (i) differences in the ratedependent parameters governing shear banding at $300 \mathrm{~K}$ compared to $77 \mathrm{~K}$, and (ii) a strongly time- and temperaturedependent process active at $77 \mathrm{~K}$ and low strain rates.

\section{Discussion}

The above experimental observations allow an interpretation of the shear band mechanism. Generally, two basic models were described in the past. Both of these rely on a change in the rheological properties of the material, causing a decrease in the viscosity within the shear band due either to adiabatic heating ${ }^{20,26)}$ (which allows the material in the vicinity of shear bands to experience a strong increase in temperature), or dilatation of the atomic structure (e.g. generation of free volume). ${ }^{13,47-49)}$ These explanations were motivated on the one hand by the observation of vein patterns (i.e. viscous flow features) on the fracture surfaces and their comparison with flow patterns generated by grease when removed from a glass plate, and on the other by the differential etching of shear bands. ${ }^{19,50)}$ Temperature increase and a possible drop in the viscosity within shear bands before fracture are still hotly debated. ${ }^{36-38,51,52)}$ However, a significant temperature increase at the moment of fracture leading to viscous flow features on the fracture surfaces seems to be generally accepted. Observations such as deformation-induced nanocrystallization, ${ }^{9,10,12,53)}$ and voids and vacancy cluster formations ${ }^{54-56)}$ find explanations in temperature rises (adiabatic heating) but may also be interpreted as an effect of dilatation and generation of free volume. In agreement with this thinking is the computational and experimental evidence for the Mohr-Coulomb yield criterion, where a stress perpendicular to the shear plane occurs. ${ }^{23,57)} \mathrm{A}$ combination of the two models can also be envisioned where a shear event is triggered by a localized temperature increase followed by rapid quenching, which would then result in a change in the atomic structure, i.e. generation of excess free volume. Strong experimental evidence for such heat excursions has recently been provided by local melting of tin in the vicinity of shear bands. ${ }^{37)}$

In the following we attempt to rationalize our results by adapting these two major models, irrespective of the cause of a drop in viscosity (i.e. adiabatic heating and/or shear dilatation). We also highlight the phenomenological similarities of our findings with the Portevin-Le Châtelier effect and propose an extension of the current view of STZs as active sites of strain by including the concept of structural relaxation as responsible for the occurrence of serrated flow.

A typical stress-strain diagram of an amorphous metal tested at room temperature like the one shown in Fig. 2(a) demonstrates that plastic deformation is accompanied by serrated flow, in which a stress drop takes place after reaching a local elastic stress maximum. This acquired elastic strain energy is suddenly released (within 10 to $100 \mathrm{~ms}$ ) and transforms into strain via the formation of a shear band. Note that similar magnitudes and necessary times recorded for single stress drops/times for a displacement-burst have been reported previously for compression ${ }^{36,58)}$ and nanoindentation $^{28,30)}$ tests on various bulk metallic glasses tested at room temperature. In addition, we observed a temperature sensitivity to the speed of the shear banding process: the time needed for a stress drop to initiate and arrest increases with decreasing temperature. At $195 \mathrm{~K}$ this process requires 0.5 to $2 \mathrm{sec}$ ! If these events are fast enough the energy release may generate a heat burst such that the viscosity of the material is lowered to a level promoting shearing along these zones. Since shear displacement (or stress drop) times in the nanosecond range must be reached to increase the temperature within the shear band sufficiently, ${ }^{37,38)}$ a decrease in the viscosity by adiabatic heating alone is questionable for the temperatures and times measured in our experiments, in particular at lower temperatures and strain rates.

Alternatively, atomic dilatation caused by the large stress field may result in a viscosity decrease and promote the stress drops and sliding. Here the shear rate first increases during sliding due to the drop in viscosity but ceases as soon as the energy supplied to keep the viscosity low is exhausted. At this point, the shear band will be arrested. Consequently the free volume generated during deformation is 'frozen in' and causes a localized softening of the material which is macroscopically detected as a shear band. If no geometrical changes in the sample occur (i.e. change in stress gradients), a successive shear event is expected to follow the same path, which is indeed the case in metallic glasses. ${ }^{19,20,33,35)}$ Thus, a continuous propagation of microscopic shear events (i.e. STZs) increases the free volume or changes the atomic configuration within the shear bands, resulting in a lower density structure compared to the undeformed material. This 
has been verified by various experiments. ${ }^{54,56,59)}$ The increase in the stress drop amplitude with increasing strain (Fig. 2(a)) can be rationalized with this explanation. At low enough testing temperatures, where no serrations occur, fewer changes in the atomic structure within the shear bands can occur in the course of deformation due to reduced atomic mobility, i.e. $m$ is positive and remains constant with $\varepsilon$ (Fig. 4).

From these considerations, however, it is not obvious why higher stresses can be reached at lower strain rates, i.e. why $m$ is negative at room temperature and why it decreases with $\varepsilon$. We recently found an analogy to this behavior in the DSA mechanism known in crystalline solid solution metals. ${ }^{35,40)}$ In the DSA mechanism, diffusion of the solvent atoms along mobile dislocations increases their resistance to glide such that with longer waiting times or slower strain rates dislocations are more effectively pinned. ${ }^{41,42)}$ In bulk metallic glasses, where the carriers of plastic deformation are believed to be clusters of a few tens of atoms (STZs), ${ }^{13-15,40)}$ the continuous alteration of the atomic shear band structure during the reactivation of a pre-existing shear band also changes the ability of the atoms to relax towards a state of lower energy (i.e., a structure closer to that of the undeformed material) after the shear event has taken place. But the more time given (i.e., the lower the strain rate), the more effective are relaxation processes in changing the atomic structure to a more stable one. This process can be understood as annihilation of free volume, therefore causing a hardening of the area in the vicinity of the shear band. Hence, at slower strain rates more time is available for the relaxation to occur between successive stress drops, so that higher stresses can be reached and produce a negative SRS. At room temperature the SRS decreases with $\varepsilon$ due to the continuous alteration of the shear bands, which increases the driving force for the structural relaxation process. This process also explains observations of decreasing stress drop magnitude with increasing strain rate (see also Ref. 35), the decrease and disappearance of pop-ins during nanoindentation tests with increasing loading rate, ${ }^{28,31,32}$ and the transition from negative to positive values of $m$ at room temperature and $195 \mathrm{~K}$ at the highest $\dot{\varepsilon}$ (Fig. 5). It is, however not clear as to whether the disappearance of serrations and positive SRS measured at room temperature and $195 \mathrm{~K}$ for higher $\dot{\varepsilon}$ is additionally influenced by the nucleation rate of STZs, as proposed by Schuh et al. ${ }^{31)}$ A simple estimate of the shear displacement per propagation time (i.e. speed of shear band) measured at low $\dot{\varepsilon}$ results in the same magnitude $(\sim 0.2 \mu \mathrm{m} /$ $\mathrm{ms})$ as the fastest cross-head displacement reached in the tests where positive values of $m$ were measured. Hence, a kinematic effect could additionally influence the positive values of $m$ at high $\dot{\varepsilon}$.

At temperatures of 77 and $153 \mathrm{~K}$ and slow strain rates, where $m$ is positive and the flow curves are smooth, relaxation processes are hampered, such that the re-arranged atomic configuration cannot relax to a lower energy state after the shear event has taken place. In this case, no serrations occur. In addition, the fracture surfaces of samples tested at $77 \mathrm{~K}$ show viscous flow features, ${ }^{40)}$ which provides evidence that high temperatures on the order of the glass transition temperature can be reached at fracture even at
$77 \mathrm{~K}$, resulting in a decrease in the viscosity. However, whether the absence of serrations is additionally influenced by a reduced decrease in viscosity cannot be answered conclusively.

The observed temperature sensitivity of $m$ at low $\dot{\varepsilon}$ (Fig. 5) suggests that different controlling parameters are active for the shear band mechanism at $300 \mathrm{~K}$ compared to $77 \mathrm{~K}$. In addition, the magnitude and decrease in $m$ with increasing $\dot{\varepsilon}$ observed at $77 \mathrm{~K}$ suggests a strongly time- and temperaturedependent process, i.e. a diffusion-dependent mechanism that dominates the kinetic flow behavior at slow strain rates.

\section{Summary}

We have shown that the method employing cycled strain rate tests at various temperatures provides important information for describing the inhomogeneous flow mechanism(s) in metallic glasses. Increasing the strain rate or decreasing the temperature well below room temperature causes the serrations to disappear and the flow curves to become smooth. This change in the flow behavior is coupled with a change in the strain rate sensitivity (SRS) from negative to positive values. In addition, a decrease in the SRS with increasing strain is observed at $300 \mathrm{~K}$, but not at $77 \mathrm{~K}$. Thorough analysis of the stress drops indicates a decrease in magnitude with increasing strain rate and decreasing temperature, while the speed of the stress drop process increases with increasing strain and temperature.

We have further shown that a concept based on the occurrence of a structural relaxation process during shear banding, in conjunction with the common understanding of shear transformation zones, accords well with the experimental findings and with knowledge of the dependence of serrated and non-serrated flow on strain, strain rate and temperature.

\section{Acknowledgements}

We thank Christian Wegmann for his help in the data analysis of stress drops. This work was supported by the Swiss National Science Foundation under Grant No. 200021105647 (F.H.D.T.) and Grant No. 200021-108071 (A.D.).

\section{REFERENCES}

1) W. L. Johnson: MRS Bull. 24 (1999) 42.

2) A. Inoue: Acta Mater. 48 (2000) 279.

3) J. F. Löffler: Intermetall. 11 (2003) 529.

4) A. L. Greer: Science 267 (1995) 1947.

5) J. Schroers and W. L. Johnson: Phys. Rev. Lett. 93 (2004) 255506.

6) A. Inoue, W. Zhang, T. Tsurui, A. R. Yavari and A. L. Greer: Phil. Mag. Lett. 85 (2005) 221.

7) J. Das, M. B. Tang, K. B. Kim, R. Theissmann, F. Baier, W. H. Wang and J. Eckert: Phys. Rev. Lett. 94 (2005) 205501.

8) Z. W. Zhu, H. F. Zhang, W. S. Sun, B. Z. Ding and Z. Q. Hu: Scripta Mater. 54 (2006) 1145.

9) J. Saida, A. D. H. Setyawan, H. Kato and A. Inoue: Appl. Phys. Lett. 87 (2005) 151907.

10) S.-W. Lee, M.-Y. Huh, E. Fleury and J.-C. Lee: Acta Mater. 54 (2006) 349.

11) J. J. Lewandowski, W. H. Wang, A. L. Greer: Phil. Mag. 85 (2005) 77.

12) M. Chen, A. Inoue, W. Zhang and T. Sakurai: Phys. Rev. Lett. 96 
(2006) 245502.

13) A. S. Argon: Acta Metall. 27 (1979) 47.

14) W. L. Johnson, K. Samwer: Phys. Rev. Lett. 95 (2005) 195501.

15) S. G. Mayr: Phys. Rev. Lett. 97 (2006) 195501.

16) Y. F. Shi and M. L. Falk: Phys. Rev. B 73 (2006) 214201.

17) Q. K. Li and M. Li: Appl. Phys. Lett. 88 (2006) 241903.

18) N. P. Bailey, J. Schiotz and K. W. Jacobsen: Phys. Rev. B 73 (2006) 064108.

19) C. A. Pampillo and H. S. Chen: Mat. Sci. Eng. 13 (1974) 181.

20) H. S. Chen: Scripta Mater. 7 (1973) 931.

21) H. Kimura and T. Masumoto: Acta Metall. 31 (1983) 231.

22) H. Kimura and T. Masumoto: Acta Metall. 28 (1980) 1663.

23) C. A. Schuh and A. C. Lund: Nature Mater. 2 (2003) 449.

24) D. B. Miracle: Nature Mater. 3 (2004) 697.

25) C. Fan, P. K. Liaw, V. Haas, J. J. Wall, H. Choo, A. Inoue and C. T. Liu: Phys. Rev. B 74 (2006) 014205.

26) H. J. Leamy, H. S. Chen and T. T. Wang: Met. Trans. 3 (1972) 699.

27) Yu. I. Golovin, V. I. Ivolgin, V. A. Khonik, K. Kitagawa and A. I. Tyurin: Scripta Mater. 45 (2001) 947.

28) C. A. Schuh and T. G. Nieh: Acta Mater. 51 (2003) 87.

29) W. H. Jiang and M. Atzmon: J. Mater. Res. 18 (2003) 755.

30) C. A. Schuh, A. S. Argon, T. G. Nieh and J. Wadsworth: Phil. Mag. A 83 (2003) 2585.

31) C. A. Schuh, A. C. Lund and T. G. Nieh: Acta Mater. 52 (2004) 5879.

32) B. Moser, J. Kuebler, H. Meinhard, W. Muster and J. Michler: Adv. Eng. Mater. 7 (2005) 388.

33) B. Moser, J. F. Löffler and J. Michler: Phil. Mag. 86 (2006) 5715.

34) T. Mukai, T. G. Nieh, Y. Kawamura, A. Inoue and K. Higashi: Intermetall. 10 (2002) 1071.

35) F. H. Dalla Torre, A. Dubach, M. E. Siegrist and J. F. Löffler: Appl. Phys. Lett. 89 (2006) 091918.

36) W. J. Wright, R. B. Schwarz and W. D. Nix: Mater. Sci. Eng. A 319321 (2001) 229.
37) J. J. Lewandowski and A. L. Greer: Nature Mater. 5 (2006) 15.

38) T. C. Hufnagel, T. Jiao, T., Y. Li, Y., L.-Q. Xing and K. T. Ramesh: J. Mater. Res. 17 (2002) 1441.

39) H. Chen, Y. He, G. J. Shiflet and S. J. Poon: Nature 367 (1994) 541.

40) A. Dubach, F. H. Dalla Torre and J. F. Löffler: Phil. Mag. Lett. (2007), in press.

41) R. A. Mulford and U. F. Kocks: Acta Metall. 27 (1979) 1125.

42) J. M. Robinson and M. P. Shaw: Internat. Mater. Rev. 39 (1994) 113.

43) A. Portevin and F. Le Châtelier: CR. Acad. Sci. 176 (1923) 507.

44) E. Pink and A. Grinberg: Acta Metall. 30 (1982) 2153.

45) S. Venkadesan, P. Rodriguez, K. A. Padmanabhan, P. V. Sivaprasad and C. Phaniraj: Mater. Sci. Eng. A 154 (1992) 69.

46) P. Hähner, A. Ziegenbein, E. Rizzi and H. Neuhäuser: Phys. Rev. B 65 (2002) 134109.

47) M. H. Cohen and D. Turnbull: J. Chem. Phys. 31 (1959) 1164.

48) M. H. Cohen and D. Turnbull: J. Chem. Phys. 52 (1970) 3038.

49) F. Spaepen: Acta Metall. 25 (1977) 407.

50) T. Masumoto and M. Maddin: Mater. Sci. Eng. 19 (1975) 1.

51) C. J. Gilbert, J. W. Ager III, V. Schroeder, R. O. Ritchie, J. P. Lloyd and J. R. Graham: Appl. Phys. Lett. 74 (1999) 3809.

52) B. Yang, M. L. Morrison, P. K. Liaw, R. A. Buchanan, G. Wang, C. T. Liu and M. Denda: Appl. Phys. Lett. 86 (2005) 141904.

53) J.-J. Kim, Y. Choi, S. Suresh and A. S. Argon: Science 295 (2002) 654.

54) K. M. Flores, D. Suh, R. Howell, P. Asoka-Kumar, P. A. Sterne and R. H. Dauskardt: Mater. Trans. JIM 42 (2001) 619.

55) J. Li, F. Spaepen and T. C. Hufnagel: Phil. Mag. A 82 (2002) 2623.

56) J. Li, Z. L. Wang and T. C. Hufnagel: Phys. Rev. B 65 (2002) 144201.

57) P. E. Donvan: Acta Metall. 37 (1989) 445.

58) W. H. Jiang, F. X. Liu, D. C. Qiao, H. Choo and P. K. Liaw: J. Mater. Res. 21 (2006) 1570

59) A. R. Yavari, A. Le Moulec, A. Inoue, N. Nishiyama, N. Lupu, E. Matsubara, W. J. Botta, G. Vaughan, M. Di Michiel and A. Kvick: Acta Mater. 53 (2005) 1611. 